\title{
Ventricular Flutter by ECG Finding
}

National Cancer Institute

\section{Source}

National Cancer Institute. Ventricular Flutter by ECG Finding. NCI Thesaurus. Code

C111115.

A ventricular tachyarrhythmia characterized by a high ventricular rate (180 and 250 beats per minute) with a regular rhythm. The electrocardiogram shows large oscillating sine wave-like complexes occurring as a result of QRS complexes and T waves being merged. The $\mathrm{P}$ wave is not visible. 